ff. mitgetheilten Bestimmungen für Ermittelung der persönlichen Gleichungen näher betrachtet.

Im verflossenen Sommer ist die Länge von Strassburg durch das Königl. preussische geodätische Institut relativ zu Berlin, Bonn, Genf und Mannheim bestimmt.

Für eine Bestimmung der Breite in aller Schärfo fehlten bislang die geeigneten Instrumente oder auch die geeignete Aufstellung. Immerhin wird es von Interesse sein, hier einige unabhängige Bestimmungen anzuführen, welche mit Hülfe von Spiegelsextanten unter Anwendung der Gauss'schen Methode der Beobachtung gleicher, aber unbeknnnter Höhe, von drei
Sternen erhalten wurden. Dabei wurde die Methode insofern erweitert, als 5 oder 7 Höhen in der Nähe der gleichen Hölıe gemessen wurden, die mittelst der Differenzialquotienten der Höhenänderung nach der Zeit auf die gleiche Höhe gebracht und aus ihnen das Mittel genommen wurde. $\mathrm{Da}$ der Thurm der Akademic für Aufstellung eines Queksilberhorizontes nicht geeignet ist, so sind die nachstehenden Beobachtungen auf einem der Akademic gegenüber befindlichen städtischen Platze angestellt, der 2".47 nördlicher liegt, als die Mitte des Thurmes Sextant von Troughton, Beobachter Hartwig.

1875 Febr. 26. $\varphi=48^{\circ} 34^{\prime} 50^{\prime \prime} .0$ “ Tauri, " Leonis, $\beta$ Ursae min.

" April 20. 56.6 a Cygni, a Bootis, “ Ursae min.

Sextant von Baumann, Beobachter Fuchs.

1876 Juli 21. $\psi=483457.4$ ^Coronae, Aquilae, $\propto$ Ursae min.

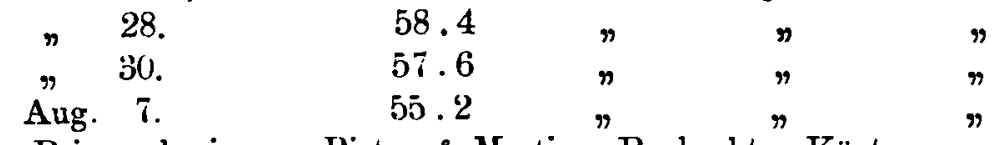

Prismenkreis von Pistor \& Martins, Beobachter Küstner.

1876 Aug. 11. $y=483455.5$ a Andromedac, « Lyrae, a Ursae min.

Die Sternörter wurden dem Nautical Almanac Uebereinstimmung zeigten die nach dieser Methode erentnommen. Nimmt man das Mittcl aus den sieben mittelten Ulircorrectionen, mit denen, welche durch das Bestimmungen, so ergiebt sich als Breite des Beob- Passageninstrument bestimmt wurden, so dass dieselbe achtungsplatzes $48^{\circ} 34^{\circ} 56^{\circ} .53$ und damit die Pollı̈he in allen Fällen, wo man constante Fehler (Excentricitat der Mitte der Axe des Pissageninstruments von Cau- des Sextanten, fehlerhafte Bestimmuug der Indexcorrecchoix zu : $\quad 48^{\circ} 34^{\prime} 54^{\prime \prime} .06$.

bis auf $6^{\prime \prime 2} 28$ übereinstimmend mit dem früher ermittelten Werthe. Eine ähnliche, wahrhaft überraschende tion, Biegung etc.) befürchten muss, eindringlich aufs Neue empfohlen werden kann.

Strassburg 1877 Februar 13. A. Winnecke.

\title{
Schreiben des Herrn Professor R. Wolf an den Herausgeber.
}

Die so eben in der Vierteljahrsschrift der Zürcherischen Naturforschenden Gesellschaft erschienene $\mathrm{Nr}$. 42 meiner "Astronomischen Mittheilungen" enthält in erster Linie die Uebersicht der Sonnenfleckenbeobachtungen im Jahre 1876 und die Angaben über die mittlere Relativzahl des Jahres, die daraus berechneten magnetischen Variationen, etc. worüber bereits in den Astronomischen-Nachrichten referirt worden ist. Sodann giebt sie eine Tafel der monatlichen Relativaahlen von 1749-1876 wodurch nun wohl der Grang des Fleckenphänomens für diese lange Periode so gut repräsentirt wird, als es überhaupt möglich ist. Nacher folgt die etwas revidirte Tafel sämmtlicher Epochen ‘für Max. und Min. seit Entdeckung der Sonnenflecken,) - ferner die nun zum ersten Male ausgemittelte vollständige mittlere Sonnenfleckencurve.) Im Anschlusse werden einige vorlaufige Vorgleichungen zwischen den mittleren und den einzclnen walıren Curven angestellt. - Die Wahrscheinlichkeit einer grösseren Sonnenfleckenperiode von circa 178 Jahren dargelegt, - und zum Schlusse folgt eine Fortsetzung der Sonnenfleckenliteratur von Nr. $344-351$.
Zürich 1877 III 9.
R. Wolf. 